\title{
YM155 exerts a growth inhibitory effect on human osteosarcoma in vitro and in vivo
}

\author{
ZHUO ZHANG ${ }^{1}$, LIANJUN MA ${ }^{1}$ and JINCHENG WANG ${ }^{2}$ \\ ${ }^{1}$ Department of Orthopedics, China-Japan Union Hospital of Jilin University, Changchun, Jilin 130033; \\ ${ }^{2}$ Department of Orthopedics, The Second Hospital of Jilin University, Changchun, Jilin 130042, P.R. China
}

Received April 24, 2015; Accepted June 3, 2015

DOI: $10.3892 /$ or.2015.4067

\begin{abstract}
YM155, a novel small-molecule inhibitor of survivin, is known to exert antitumor effects on various cancers, including breast, prostate and lung cancer. However, there are few studies describing the inhibitory effect of YM155 on human osteosarcoma (OS) which highly expresses survivin. Here, we tested the effects of YM155 on OS cells by several in vitro experiments. It was found that YM155 inhibited cell proliferation, colony formation, migration and invasion, induced cell apoptosis, as well as increased caspase-3, -8 and -9 activity in the OS cell lines in a dose-dependent manner. We also found that YM155 suppressed Mcl-1 and survivin expression without affecting the expression of anti-apoptotic proteins X-linked inhibitor of apoptosis (XIAP) and Bcl-2. In addition, YM155 decreased phosphoinositide 3-kinase (PI3K) and AKT expression without effecting total PI3K and AKT in the OS cell lines, which contributed to suppression of OS tumor growth at least in part. In addition, YM155 also suppressed tumor growth in vivo, reducing the size of OS MG63 cell xenografts. Taken together, the findings revealed that YM155 suppresses the tumor growth of OS in vitro and in vivo, suggesting that YM155 has potential as a therapeutic agent for the treatment of OS.
\end{abstract}

\section{Introduction}

Osteosarcoma (OS) is the leading primary malignant bone tumor in children and adolescence $(1,2)$. Pulmonary metastases, central presentation and local non-resectable relapse are common in the majority of patients, contributing to a high mortality rate (3). Although advancements in surgical techniques, radiation and chemotherapy strategies have increased the local control of OS, the overall survival of OS patients has still remained relatively constant for over two decades $(4,5)$. Therefore, novel therapeutic agents are desperately needed to further improve the prognosis of OS patients.

Correspondence to: Professor Jincheng Wang, Department of Orthopedics, The Second Hospital of Jilin University, 218 Ziqiang Street, Nanguan, Changchun, Jilin 130042, P.R. China

E-mail: wangjincheng5413@sina.cn

Key words: osteosarcoma, YM155, survivin, PI3K/AKT
Survivin, a member of the inhibitors of apoptosis (IAP) protein family, encoded by the BIRC5 gene, is highly expressed in various types of cancer cells while it is not expressed or expressed at a substantially lower level in their normal tissue counterparts (6). Furthermore, the expression level of survivin has been found to correlate with poor prognosis in gastric (7), colorectal (8), non-small cell lung (9), breast (10), pancreatic (11), ovarian (12), prostate cancers (13), and melanoma (14). Functionally, survivin has been shown to counteract apoptosis induction upstream of effector caspases (15) and to play a critical role in the regulation of cell division by inducing exit from G1 checkpoint arrest and subsequent entry into the $S$ phase (16). In addition, survivin also plays an essential role in cell proliferation by regulating spindle assembly and microtubule attachment to the kinetochore as a member of the chromosomal passenger complex $(17,18)$. Growing evidence has demonstrated that inhibition of survivin expression using antisense oligonucleotides, small-molecule antagonists, or small interfering RNA (siRNA) suppresses tumor cell proliferation and invasion, induces cell apoptosis, consequently suppressing tumor growth (13-18).

Recently, YM155, a novel small-molecule inhibitor of survivin, was identified by cell-based high throughput screening using a survivin promoter luciferase assay (19). In vitro, YM155 exerts potent antitumor activity in various types of cancer cells including non-small cell lung cancer, non-Hodgkin's lymphoma, melanoma and hormone-refractory prostate cancer (20-24). In vivo, YM155 has also shown anticancer efficacy in lung and prostate cancer in xenograft models $(21,25)$. In addition, YM155 has been evaluated in phase II clinical trials in patients with advanced refractory non-small cell lung carcinoma and unresectable melanoma $(26,27)$. But little research has been carried out on the effects of YM155 against OS, and no tools are available to guide the use of YM155 in the treatment of OS.

Therefore, in the present study, we evaluated the anticancer effects of YM155 and the underlying molecular mechanism in OS cell lines. We also investigated the anticancer effects of YM155 on OS cell line MG63 xenografts.

\section{Materials and methods}

Reagents and antibodies. The small-molecule survivin inhibitor YM155 (Fig. 1A) was purchased from Selleck Chemicals 
(Houston, TX, USA). For the in vitro studies, YM155 was dissolved in dimethyl sulfoxide (DMSO) and stored at $-20^{\circ} \mathrm{C}$.

Primary antibodies against the following proteins were used for western blot analyses: survivin (monoclonal; Abcam, Cambridge, MA, USA), GAPDH (Santa Cruz Biotechnology, Santa Cruz, CA, USA), XIAP (monoclonal; BD Biosciences, San Jose, CA, USA), phosphoinositide 3-kinase (PI3K), phospho (p)-PI3K (Tyr458), AKT, p-AKT (Ser473), Bcl-2 and Mcl-1 (Cell Signaling Technology, Danvers, MA, USA).

Cell culture. Two human OS cell lines, MG63 and Saos-2, were purchased from the Cell Bank of the Type Culture Collection of the Chinese Academy of Sciences (Shanghai, China). All cells were grown in Dulbecco's modified Eagle's medium (DMEM) supplemented with $10 \%$ fetal bovine serum (FBS) (both from Gibco-BRL, Grand Island, NY, USA), 2 mM glutamine, $100 \mathrm{U} / \mathrm{ml}$ penicillin and $100 \mathrm{mg} / \mathrm{ml}$ streptomycin at $37^{\circ} \mathrm{C}$ in a $5 \% \mathrm{CO}_{2}$ atmosphere and at $95 \%$ humidity.

Cell viability and colony formation. The MTT assay was used to determine the effect of different concentrations of YM155 on the proliferation of cells. Briefly, cells grown in monolayers were collected and dispensed in 96-well culture plates in $100 \mu \mathrm{l}$ of DMEM at a concentration of $5 \times 10^{3}$ cells/ well. After $24 \mathrm{~h}$, differential concentrations of YM155 (0, 1, 10 and $100 \mathrm{nM}$ ) were added to the cells. Seventy-two hours after treatment, cell proliferation was measured as described previously (28). This assay was performed in triplicate.

The effect of YM155 on cell colony formation was assessed using a clonogenic assay. Briefly, $1.0 \times 10^{3}$ cells were plated in 6 -well plates in growth medium, and after overnight attachment, the cells were treated with different concentrations of YM155 (0, 1, 10 and $100 \mathrm{nM})$ for $24 \mathrm{~h}$. The cells were then washed with PBS and allowed to grow for 14 days in drug-free conditions, and the medium was replaced every 3 days. The colonies were fixed with $4 \%$ paraformaldehyde for $20 \mathrm{~min}$ and stained with $1 \%$ crystal violet for $10 \mathrm{~min}$. Colonies containing $>50$ cells were counted.

Cell apoptosis. The effect of YM155 on cell apoptosis was determined by flow cytometry. Briefly, 5.0x $10^{5}$ cells were plated in 60-mm dishes and treated with different concentrations of YM155 (0, 1, 10 and $100 \mathrm{nM})$ for $24 \mathrm{~h}$. After treatment, the cells were stained with Annexin V (Molecular Probes) and propidium iodide (Sigma-Aldrich, St. Louis, MO, USA) and analyzed using flow cytometry (BD Biosciences, Mansfield, MA, USA). The apoptosis ratio was analyzed by CellQuest software (BD Biosciences).

In addition, caspase- $3,-8$ and -9 activity was detected as an additional indicator of apoptosis using Caspases Colorimetric Protease Assay kits (Millipore Corporation, Billerica, MA, USA) as previously described (28). The relative caspase-3, - 8 and -9 activity of the control blank group was referred to as 100 .

Cell migration and invasion assays. The migration assay was performed with Transwell inserts with $8.0-\mathrm{mm}$ pore size membrane (Corning, Tewksbury, MA, USA). For the invasion assay, the previously mentioned inserts were pre-coated with Matrigel matrix (BD Science, Sparks, MD, USA). The cells $\left(1 \times 10^{5}\right)$ were resuspended in serum-free medium and seeded into the upper chamber. DMEM with $10 \%$ FBS was added to the lower chamber to serve as a chemoattractant. After incubation at $37^{\circ} \mathrm{C}$ for $48 \mathrm{~h}$, the migrated and invaded cells present on the lower side of the membrane were fixed with $70 \%$ ethanol for $30 \mathrm{~min}$ and stained with $0.2 \%$ crystal violet for $10 \mathrm{~min}$. The number of migrated and invaded cells was counted in 5 randomly selected fields by an inverted microscope (Olympus, Tokyo, Japan).

Western blot assays. The cells were lysed in lysis solution (Cell Signaling Technology) supplemented with sodium fluoride (10 M; Fisher) and phenylmethylsulfonyl fluoride (100 g/ml; Sigma-Aldrich) for $30 \mathrm{~min}$. Lysates were centrifuged at $14,000 \mathrm{x} \mathrm{g}$ for $10 \mathrm{~min}$, and the total protein concentration was determinated using the BCA assay kit (Sigma-Aldrich). Each $20 \mu \mathrm{g}$ of sample was fractionated on either 8 or $12 \%$ SDS-polyacrylamide gel, and the separated proteins were transferred to nitrocellulose membranes (Bio-Rad, Munich, Germany). The membranes were blocked with 5\% non-fat dry milk for $2 \mathrm{~h}$ and incubated with the previously mentioned primary antibody overnight at $4^{\circ} \mathrm{C}$, followed by incubation with horseradish peroxidase-conjugated goat anti-mouse IgG (Santa Cruz Biotechnology) for $2 \mathrm{~h}$ at room temperature. Protein bands were visualized with enhanced chemiluminescence reagent (ECL; Amersham, GE Healthcare, Velizy-Villacoublay, France). Protein loading was normalized by stripping the blots and then reprobing with the anti-GAPDH antibody.

Xenograft model. Five-week-old male BALB/c nude mice (5-6 weeks old) were purchased from the Experimental Animal Center of Changchun Biological Institute (Changchun, China), and maintained under specific pathogen-free (SPF) conditions. This study was approved by the Animal Ethics Committee of Jilin University (Changchun, China).

Tumors were established by injecting $2 \times 10^{6}$ MG63 cells subcutaneously into the right flank of the mice. When subcutaneous tumors reached a size of $100 \mathrm{~mm}^{3}$, the xenografted animals were randomly divided into vehicle and YM155 (10 mg/kg) groups. YM155 was subcutaneously administered as a 3-day continuous infusion per week for 4 weeks using an implanted micro-osmotic pump (Alzet Model 1003D; Durect, Cupertino, CA, USA) as previously described (19). Tumors were measured every 5 days with calipers, and the volume $\left(\mathrm{mm}^{3}\right)$ was calculated according to the following formula: $[\pi / 6$ $\mathrm{x}$ length $\mathrm{x}$ width $\mathrm{x}$ height]. Thirty days after inoculation, the mice were sacrificed, and tumors were stripped and weighed, and analyzed for survivin expression by western blot analysis.

Statistical analysis. All data are expressed as mean \pm standard deviation (SD). Statistical analysis between two samples was performed using the Student's t-test and for more than two groups, analysis was performed using one-way ANOVA followed by a Tukey's post hoc test using GraphPad Prism 5.0 software (GraphPad Software, San Diego, CA, USA). Significance was set at $\mathrm{P}<0.05$.

\section{Results}

YM155 inhibits survivin expression in OS cells. Recently, the small-molecule survivin inhibitor YM155 (Fig. 1A) has 
A

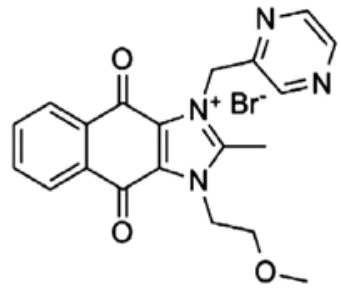

B

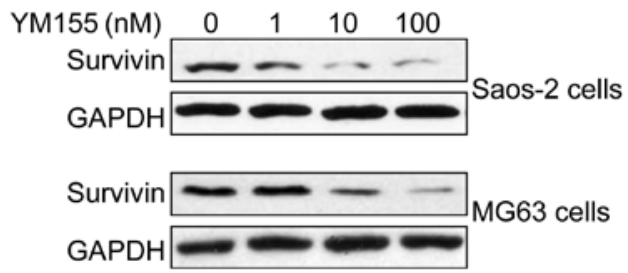

Figure 1. YM155 inhibits survivin protein expression in OS cell lines in a dose-dependent manner. (A) Chemical structure of YM155. (B) Survivin expression was examined in MG63 and Saos-2 cells after treatment with different doses of YM155 (0, 1, 10 and $100 \mathrm{nM})$. GAPDH was used as a control. OS, osteosarcoma.

been shown to inhibit survivin expression in various types of cancers (13-18). However, the effect of YM155 on survivin expression in OS cells has been not reported to date. Next, we examined whether YM155 inhibits survivin expression at the protein level in the OS cell lines by western blot analysis $24 \mathrm{~h}$ after treatment with various doses of YM155 (0, $1,10$ and $100 \mathrm{nM})$. The results of the western blot analysis demonstrated that YM155 significantly inhibited survivin expression in the MG63 and Saos-2 cells in a dose-dependent manner (Fig. 1B).

YM155 inhibits cell proliferation, colony formation, migration and invasion in OS cells. To evaluate the effect of YM155 on the cell proliferation of OS cells, MG63 and Saos-2 cells were treated with different concentrations of YM155 $(0,1,10$ and $100 \mathrm{nM})$ for 1-3 days, then an MTT assay was performed. The result showed that YM155 inhibited the cell proliferation of MG63 and Saos-2 cells in a dose-dependent manner (Fig. 2A).

Next, the effects of YM155 on the cell colony formation of OS cells were also determined. As shown Fig. 2B, YM155 obviously decreased colony formation number of OS cells (MG63 and Saos-2) in a dose-dependent manner $(\mathrm{P}<0.05)$.

We also investigate whether YM155 affects cell migration and invasion of the OS cells by Transwell assay after treatment with YM155. It was found that YM155 significantly suppressed migration (Fig. 2C) and invasion (Fig. 2D) in the MG63 and Saos- 2 cells in a dose-dependent manner $(\mathrm{P}<0.05)$.

YM155 induces cell apoptosis and increases caspase activity in OS cells. To examine the effect of YM155 on cell apoptosis, we performed apoptosis assays using the Annexin V-FITC/PI staining method. Our result showed that YM155 significantly induced apoptosis in the MG63 and Saos-2 cells in a dose-dependent manner (Fig. 3A, P<0.05).

In addition, we also evaluated the effect of YM155 on caspase activity in the OS cells as previously described (28). YM155 significantly increased caspase-3, -8 and -9 activity in the MG63 and Saos-2 cells in a dose-dependent manner (Fig. 3B-D, P<0.05).

YM155 affects the expression of anti-apoptotic proteins in OS cells. To determine the potential mechanism of YM155-induced cell apoptosis of OS cells, we investigated the effects of YM155 on the expression of anti-apoptotic proteins, such as XIAP, Bcl-2 and Mcl-1 in the OS cell lines (Fig. 4). Western blot assay showed that YM155 significantly decreased Mcl-1 protein expression in the OS cell lines in a dose-dependent manner. There was no change in Bcl-2 or XIAP levels in the OS cell lines after treatment with YM155.
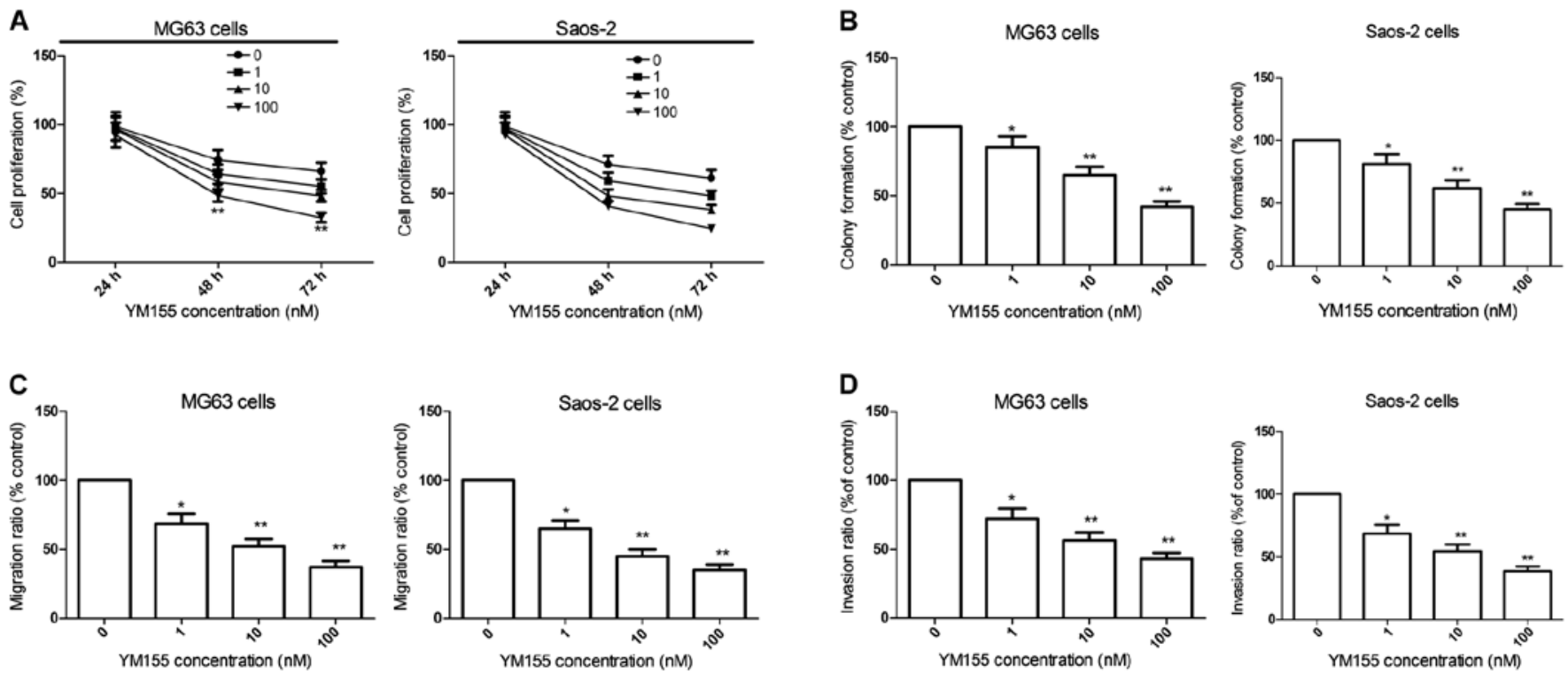

Figure 2. YM155 inhibits cell proliferation, colony formation, migration and invasion of OS cells in a dose-dependent manner. (A) Cell proliferation, (B) colony formation, (C) cell migration and (D) cell invasion of MG63 and Saos-2 cells were determined after treatment with various concentrations of YM155 (0, 1 , 10 and $100 \mathrm{nM}) .{ }^{*} \mathrm{P}<0.05,{ }^{* *} \mathrm{P}<0.01$ vs. control (untreated group). 
A
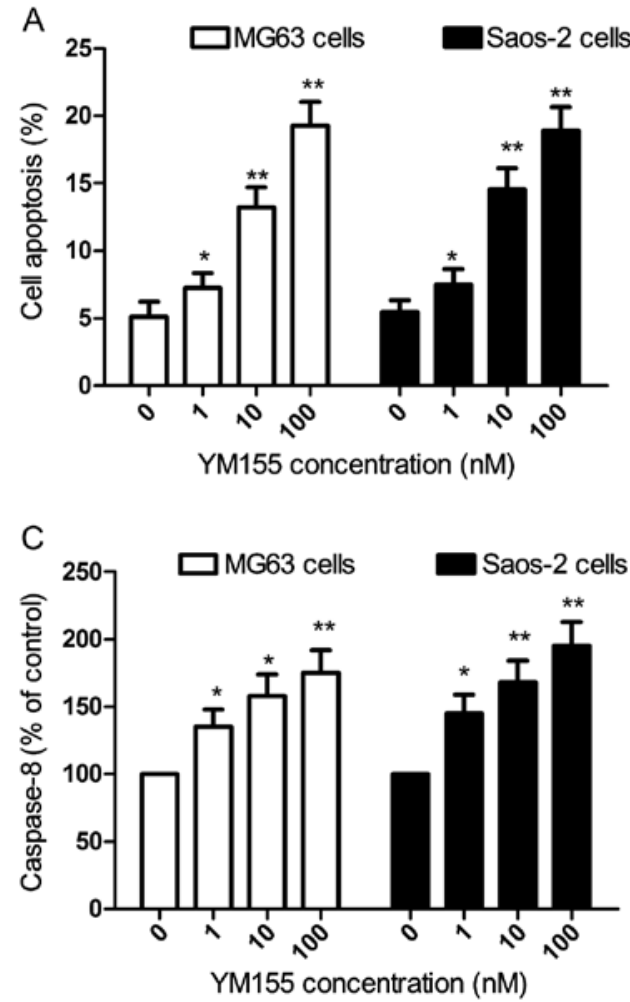
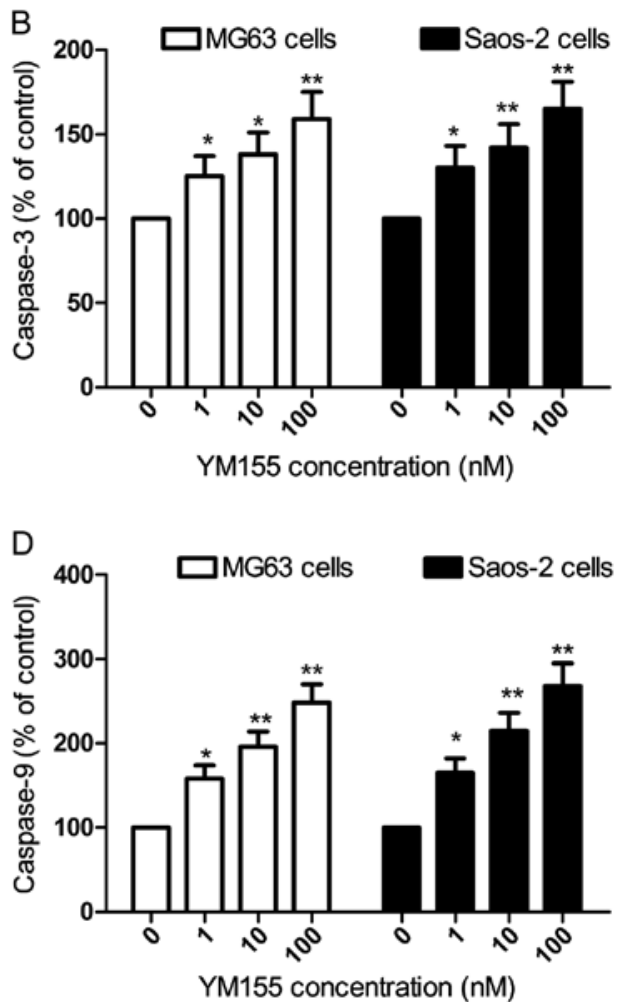

Figure 3. YM155 induces cell apoptosis and increases caspase-3, -8 and -9 activity in the OS cells in a dose-dependent manner. (A) Cell apoptosis of MG63 and Saos- 2 cells was determined by flow cytometry after treatment with various concentrations of YM155 ( $0,1,10$ and $100 \mathrm{nM})$. (B-D) Caspase-3, -8 and -9 activity was measured after treatment with various concentrations of YM155 $(0,1,10$ and $100 \mathrm{nM}) .{ }^{*} \mathrm{P}<0.05,{ }^{* *} \mathrm{P}<0.01 \mathrm{vs}$. control (untreated group). OS, osteosarcoma.
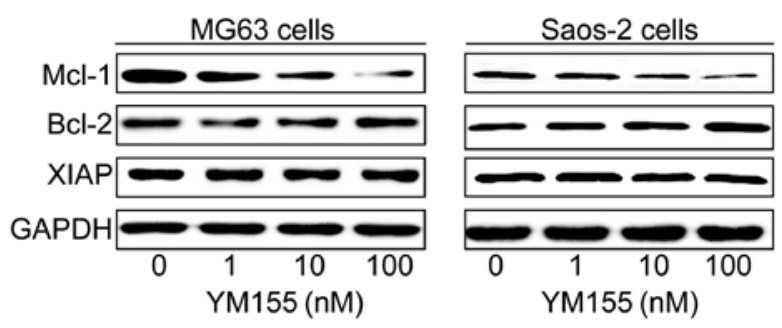

Figure 4. YM155 affects expression of anti-apoptotic proteins in the OS cells Expression of XIAP, Bcl-2 and Mcl-1 protein in OS cells was determined by western blot analysis after treatment with various concentrations of YM155 $(0,1,10$ and $100 \mathrm{nM})$. GAPDH was used as a control. OS, osteosarcoma; XIAP, $\mathrm{X}$-linked inhibitor of apoptosis protein.

YM155 affects the PI3K/AKT signaling pathway in OS cells. It has been showed that the PI3K/AKT signaling pathway plays a crucial role in cell proliferation, apoptosis, migration and invasion (29). Previous studies have shown that inhibition of PI3K and AKT downregulates survivin protein expression $(30,31)$. Here, we aimed to ascertain whether YM155 affects the expression of the PI3K/AKT signaling pathway in the OS cells. We examined PI3K, AKT, p-PI3K and p-AKT expression by western blot analysis, and found that YM155 reduced the expression of p-PI3K (Tyr458) and p-AKT (Ser473) in the OS cells, whereas the total PI3K and AKT remained unchanged (Fig. 5).

Antitumor effect of YM155 in a xenograft model. To confirm the antitumor effects of YM155 in vivo, we used a BALB/c
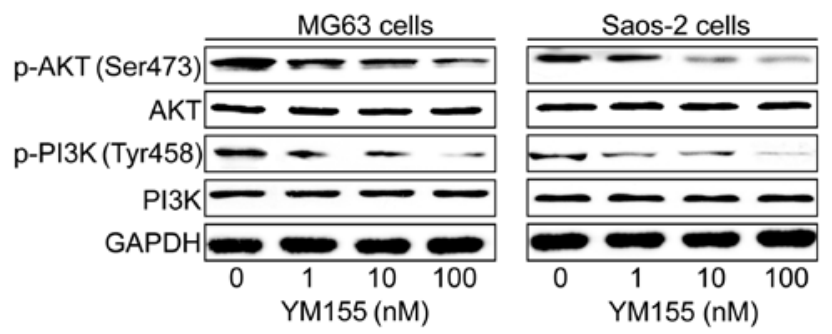

Figure 5. YM155 affects the PI3K/AKT signaling pathway in OS cells. Expression of PI3K, p-PI3K, AKT and p-AKT proteins in OS cells was determined by western blot analysis after treatment with various concentrations of YM155 (0, 1, 10 and $100 \mathrm{nM})$. GAPDH was used as a control. OS, osteosarcoma; PI3K, phosphoinositide 3-kinase.

mouse xenograft model. Animals bearing MG63 tumors were treated with YM155. Control mice were treated with saline (carrier) equivalently delivered. YM155 was well-tolerated, and it did not cause any animal mortality or induce significant decrease in body weight compared to the control group. Mice were sacrificed 30 days after treatment initiation, and tumor tissue was excised. YM155 treatment markedly abrogated tumor growth (Fig. 6A); the average size of the tumors from the control group at the study termination was $1,423 \pm 113$ vs. $624 \pm 67 \mathrm{~mm}^{3}$ in the YM155-treated group (P<0.05, Fig. 6B). Moreover, treatment with YM155 significantly reduced tumor weight compared with the control $(\mathrm{P}<0.05)$. Average tumor weights at study termination were $1.42 \pm 0.13$ and $0.62 \pm 0.10 \mathrm{~g}$ in the control and YM155 group, respectively (Fig. 6C). In addition, survivin expression in the xenograft tumors was 
A

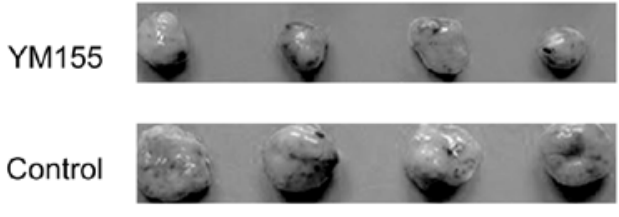

C

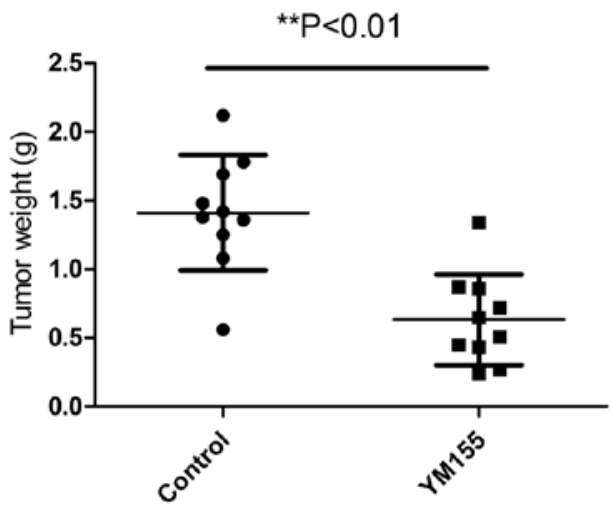

B

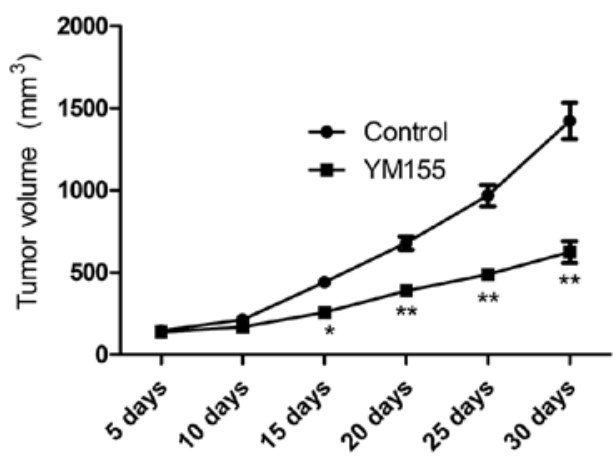

D

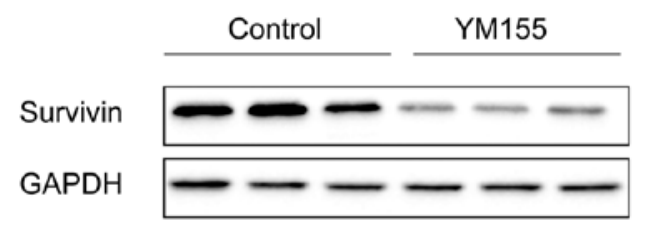

Figure 6. Antitumor activity of YM155 in BALB/c mice bearing MG63 cell-derived tumors. Animals bearing MG63 tumors were treated with YM155 $(10 \mathrm{mg} / \mathrm{kg}$ ) for 30 days. (A) Representative photomicrographs of tumors from the untreated (control) and YM155 treatment group. (B) Tumor growth curves for MG63 tumors treated with YM155 and untreated (control) group. (C) Tumor weight from untreated (control) and YM155 treatment group. (D) Survivin protein expression in tumor tissues was determined by western blot analysis. GAPDH was used as a control. ${ }^{*} \mathrm{P}<0.05,{ }^{* *} \mathrm{P}<0.01$ vs. control (untreated group).

determined by western blot analysis. The results revealed that survivin expression was downregulated in the YM155 treatment group compared to the other groups (Fig. 6D). These results suggest that YM155 markedly suppressed OS tumorigenicity in nude mice through inhibition of survivin expression.

\section{Discussion}

Survivin, a member of the inhibitor of apoptosis protein family, is involved in cell survival and regulation of mitosis in various types of cancer (6-10). Growing evidence has demonstrated that survivin suppression induces tumor cell apoptosis and enhances sensitivity to apoptosis induced by existing anticancer drugs and other apoptotic stimuli (13-18), suggesting that survivin may be a new avenue for anticancer treatment. Overexpression of survivin has been reported to be important in the development and progression of OS cells $(32,33)$. Shoeneman et al found that inhibition of survivin expression in canine OS cells by siRNA inhibited cell-cycle progression, increased apoptosis, mitotic arrest, and chemosensitivity, and cooperated with chemotherapy to obviously improve in vivo tumor control (34). Zou et al found that that in OS cells, downregulation of survivin with the use of the pSUPER-sh vector induced cell apoptosis and promoted induction of cell death by chemotherapy (35). In agreement with these results, in the present study, our results showed that inhibition of survivin expression by YM155, a small-molecule inhibitor of survivin, significantly suppressed OS growth in vitro and in vivo, suggesting that survivin is a novel target for anticancer therapy for OS.

Several therapeutic approaches for inhibiting survivin expression, such as immunotherapy or small-molecule antagonists, siRNA, either as single agents or in combination with conventional chemotherapeutic agents, are currently in clinical trials (36). YM155, identified as a novel smallmolecule survivin suppressant, has been showed to have potent anticancer activity against a broad spectrum of human cancer cell lines, such as lung, breast, prostate, ovarian and bladder cancer, and various human-derived tumor xenograft mouse models (21). However, there are few studies describing the inhibitory effect of YM155 on human OS which highly expresses survivin. Here, we tested the effects of YM155 on OS cells. The present study showed that YM155 inhibited MG63 and Saos- 2 cell proliferation and migration and invasion, as well as induced cell apoptosis and increased caspase- $3,-8$ and -9 activity, suggesting that YM155 has effective anticancer activity against $\mathrm{OS}$.

Although YM155 has been shown to have antitumor activities in vitro, in vivo and in clinical trials, the mechanism underlying human cancer susceptibility to YM155 remains to be fully elucidated. Recently, it was reported that YM155 exerts an anti-proliferative effect, inducing cell apoptosis through Mcl-1 in various cancer cell types (37). Feng et al (38) also demonstrated that YM155 exhibited robust cytotoxic activity through downregulation of survivin and Mcl-1 in human leukemia cells. Mcl-1 is a widely expressed pro-survival Bcl-2 family member. It protects cells from stimulus-triggered 
apoptosis, while inhibition of Mcl-1 usually correlates with induction of cellular apoptosis (39). Consistent with these results, the present study showed that YM155 significantly inhibited survivin and Mcl-1 protein expression in OS cell lines in a dose-dependent manner, without altering the Bcl-2 or XIAP levels in the OS cell lines, suggesting that YM155 suppresses OS growth through inhibition of survivin and Mcl-1 expression.

An increasing body of evidence has shown that the $\mathrm{PI} 3 \mathrm{~K} / \mathrm{AKT}$ pathway is frequently hyperactivated in OS and contributes to disease initiation and development, including tumorigenesis, proliferation, invasion, cell cycle progression, inhibition of apoptosis, angiogenesis, metastasis and chemoresistance $(29,40)$. Inhibition of this pathway using small-molecule compounds represents an attractive potential therapeutic approach for OS (40). Previous studies have shown that inhibition of PI3K and AKT through a PI3K or an AKT-specific inhibitor decreased the survivin protein expression in cancer cells $(30,31)$. Of note, recently a study showed that YM155 downregulates PI3K expression in human pancreatic cancer cells (41). Here, we found that YM155 reduced the expression of p-PI3K (Tyr458) and p-AKT (Ser473) expression, whereas the total PI3K and AKT remained unchanged (Fig. 5), suggesting that YM155 inhibits OS cell growth in vitro and in vivo, at least in part, via inhibiting the activation of the PI3K/AKT signaling pathway.

In summary, we present evidence that YM155 significantly inhibits OS cell proliferation, colony formation, migration and invasion, and induces cell apoptosis and increases caspase-3, -8 and -9 activities in vitro, as well as suppresses tumor growth in vivo through inhibition of survivin and Mcl-1 expression. In addition, YM155 inhibits the activation of the PI3K/AKT signaling pathway, which contributes to inhibition of OS cell survival. These findings suggest that YM155 may be a promising drug candidate for the treatment of OS.

\section{References}

1. Ottaviani G, Robert RS, Huh WW, Palla S and Jaffe N: Sociooccupational and physical outcomes more than 20 years after the diagnosis of osteosarcoma in children and adolescents: Limb salvage versus amputation. Cancer 119: 3727-3736, 2013.

2. Ottaviani G and Jaffe N: The epidemiology of osteosarcoma. Cancer Treat Res 152: 3-13, 2009.

3. Ozaki T, Flege S, Kevric M, Lindner N, Maas R, Delling G, Schwarz R, von Hochstetter AR, Salzer-Kuntschik M, Berdel WE, et al: Osteosarcoma of the pelvis: Experience of the Cooperative Osteosarcoma Study Group. J Clin Oncol 21: 334-341, 2003.

4. Chou AJ, Kleinerman ES, Krailo MD, Chen Z, Betcher DL, Healey JH, Conrad EU III, Nieder ML, Weiner MA, Wells RJ, et al; Children's Oncology Group: Addition of muramyl tripeptide to chemotherapy for patients with newly diagnosed metastatic osteosarcoma: A report from the Children's Oncology Group. Cancer 115: 5339-5348, 2009.

5. Grignani G, Palmerini E, Dileo P, Asaftei SD, D'Ambrosio L, Pignochino Y, Mercuri M, Picci P, Fagioli F, Casali PG, et al: A phase II trial of sorafenib in relapsed and unresectable high-grade osteosarcoma after failure of standard multimodal therapy: An Italian Sarcoma Group study. Ann Oncol 23: 508-516, 2012.

6. Altieri DC: Survivin, cancer networks and pathway-directed drug discovery. Nat Rev Cancer 8: 61-70, 2008.

7. Lu CD, Altieri DC and Tanigawa N: Expression of a novel antiapoptosis gene, survivin, correlated with tumor cell apoptosis and p53 accumulation in gastric carcinomas. Cancer Res 58: 1808-1812, 1998.
8. Kawasaki H, Altieri DC, Lu CD, Toyoda M, Tenjo T and Tanigawa N: Inhibition of apoptosis by survivin predicts shorter survival rates in colorectal cancer. Cancer Res 58: 5071-5074, 1998.

9. Monzó M, Rosell R, Felip E, Astudillo J, Sánchez JJ, Maestre J, Martín C, Font A, Barnadas A and Abad A: A novel anti-apoptosis gene: Re-expression of survivin messenger RNA as a prognosis marker in non-small-cell lung cancers. J Clin Oncol 17: 2100-2104, 1999.

10. Tanaka K, Iwamoto S, Gon G, Nohara T, Iwamoto $M$ and Tanigawa N: Expression of survivin and its relationship to loss of apoptosis in breast carcinomas. Clin Cancer Res 6: 127-134, 2000.

11. Liggins C, Orlicky DJ, Bloomquist LA and Gianani R: Developmentally regulated expression of Survivin in human pancreatic islets. Pediatr Dev Pathol 6: 392-397, 2003.

12. Xing J, Jia CR, Wang Y, Guo J and Cai Y: Effect of shRNA targeting survivin on ovarian cancer. J Cancer Res Clin Oncol 138: 1221-1229, 2012.

13. Krajewska M, Krajewski S, Banares S, Huang X, Turner B, Bubendorf L, Kallioniemi OP, Shabaik A, Vitiello A, Peehl D, et al: Elevated expression of inhibitor of apoptosis proteins in prostate cancer. Clin Cancer Res 9: 4914-4925, 2003.

14. McKenzie JA and Grossman D: Role of the apoptotic and mitotic regulator survivin in melanoma. Anticancer Res 32: 397-404, 2012.

15. Dohi T, Okada K, Xia F, Wilford CE, Samuel T, Welsh K, Marusawa $\mathrm{H}$, Zou $\mathrm{H}$, Armstrong $\mathrm{R}$, Matsuzawa $\mathrm{S}$, et al: An IAP-IAP complex inhibits apoptosis. J Biol Chem 279: 34087-34090, 2004.

16. Wheatley SP and McNeish IA: Survivin: A protein with dual roles in mitosis and apoptosis. Int Rev Cytol 247: 35-88, 2005.

17. Tulu US, Fagerstrom C, Ferenz NP and Wadsworth P: Molecular requirements for kinetochore-associated microtubule formation in mammalian cells. Curr Biol 16: 536-541, 2006.

18. Sampath SC, Ohi R, Leismann O, Salic A, Pozniakovski A and Funabiki H: The chromosomal passenger complex is required for chromatin-induced microtubule stabilization and spindle assembly. Cell 118: 187-202, 2004.

19. Nakahara T, Kita A, Yamanaka K, Mori M, Amino N, Takeuchi M, Tominaga F, Hatakeyama S, Kinoyama I, Matsuhisa A, et al: YM155, a novel small-molecule survivin suppressant, induces regression of established human hormone-refractory prostate tumor xenografts. Cancer Res 67: 8014-8021, 2007.

20. Kawano H, Shakushiro K, Nakata M, Kita A, Maeda A, Watanabe S, Sako K and Oku N: Antitumor efficacy and biodistribution of liposomal sepantronium bromide (YM155), a novel small-molecule survivin suppressant. Eur J Pharm Biopharm 88: 283-289, 2014.

21. Nakahara T, Kita A, Yamanaka K, Mori M, Amino N, Takeuchi M, Tominaga F, Kinoyama I, Matsuhisa A, Kudou M, et al: Broad spectrum and potent antitumor activities of YM155, a novel small-molecule survivin suppressant, in a wide variety of human cancer cell lines and xenograft models. Cancer Sci 102: 614-621, 2011.

22. Minematsu T, Iwai M, Sugimoto K, Shirai N, Nakahara T, Usui $\mathrm{T}$ and Kamimura $\mathrm{H}$ : Carrier-mediated uptake of 1-(2-methoxyethyl)-2-methyl-4,9-dioxo-3-(pyrazin-2-ylmethyl)-4,9dihydro-1H-naphtho[2,3-d]imidazolium bromide (YM155 monobromide), a novel small-molecule survivin suppressant, into human solid tumor and lymphoma cells. Drug Metab Dispos 37: 619-628, 2009.

23. Kita A, Nakahara T, Yamanaka K, Nakano K, Nakata M, Mori M, Kaneko N, Koutoku H, Izumisawa N and Sasamata M: Antitumor effects of YM155, a novel survivin suppressant, against human aggressive non-Hodgkin lymphoma. Leuk Res 35: 787-792, 2011.

24. Tolcher AW, Quinn DI, Ferrari A, Ahmann F, Giaccone G, Drake T, Keating A and de Bono JS: A phase II study of YM155, a novel small-molecule suppressor of survivin, in castration-resistant taxane-pretreated prostate cancer. Ann Oncol 4: 968-973, 2012.

25. Zhang K, Li Y, Liu W, Gao X and Zhang K: Silencing survivin expression inhibits the tumor growth of non-small-cell lung cancer cells in vitro and in vivo. Mol Med Rep 11: 639-644, 2015.

26. Giaccone G, Zatloukal P, Roubec J, Floor K, Musil J, Kuta M, van Klaveren RJ, Chaudhary S, Gunther A and Shamsili S: Multicenter phase II trial of YM155, a small-molecule suppressor of survivin, in patients with advanced, refractory, non-small-cell lung cancer. J Clin Oncol 27: 4481-4486, 2009. 
27. Lewis KD, Samlowski W, Ward J, Catlett J, Cranmer L, Kirkwood J, Lawson D, Whitman E and Gonzalez R: A multi-center phase II evaluation of the small molecule survivin suppressor YM155 in patients with unresectable stage III or IV melanoma. Invest New Drugs 29: 161-166, 2011.

28. Yang Q, Zhang S, Kang M, Dong R and Zhao J: Synergistic growth inhibition by sorafenib and cisplatin in human osteosarcoma cells. Oncol Rep 33: 2537-2544, 2015.

29. Follo MY, Manzoli L, Poli A, McCubrey JA and Cocco L: PLC and PI3K/Akt/mTOR signalling in disease and cancer. Adv Biol Regul 57: 10-16, 2015.

30. Hideshima T, Catley L, Raje N, Chauhan D, Podar K, Mitsiades C, Tai YT, Vallet S, Kiziltepe T, Ocio E, et al: Inhibition of Akt induces significant downregulation of survivin and cytotoxicity in human multiple myeloma cells. Br J Haematol 138: 783-791, 2007.

31. Siddiqa A, Long LM, Li L, Marciniak RA and Kazhdan I: Expression of HER-2 in MCF-7 breast cancer cells modulates anti-apoptotic proteins Survivin and Bcl-2 via the extracellular signal-related kinase (ERK) and phosphoinositide-3 kinase (PI3K) signalling pathways. BMC Cancer 8: 129, 2008.

32. Osaka E, Suzuki T, Osaka S, Yoshida Y, Sugita H, Asami S, Tabata K, Hemmi A, Sugitani M, Nemoto N, et al: Survivin as a prognostic factor for osteosarcoma patients. Acta Histochem Cytochem 39: 95-100, 2006.

33. Trieb K, Lehner R, Stulnig T, Sulzbacher I and Shroyer KR: Survivin expression in human osteosarcoma is a marker for survival. Eur J Surg Oncol 29: 379-382, 2003.
34. Shoeneman JK, Ehrhart EJ III, Eickhoff JC, Charles JB, Powers BE and Thamm DH: Expression and function of survivin in canine osteosarcoma. Cancer Res 72: 249-259, 2012.

35. Zou J, Gan M, Mao N, Zhu X, Shi Q and Yang H: Sensitization of osteosarcoma cell line SaOS-2 to chemotherapy by downregulating survivin. Arch Med Res 41: 162-169, 2010.

36. Church DN and Talbot DC: Survivin in solid tumors: Rationale for development of inhibitors. Curr Oncol Rep 14: 120-128, 2012.

37. Tang H, Shao H, Yu C and Hou J: Mcl-1 downregulation by YM155 contributes to its synergistic anti-tumor activities with ABT-263. Biochem Pharmacol 82: 1066-1072, 2011.

38. Feng W, Yoshida A and Ueda T: YM155 induces caspase- 8 dependent apoptosis through downregulation of survivin and Mcl-1 in human leukemia cells. Biochem Biophys Res Commun 435: 52-57, 2013.

39. Thomas LW, Lam C and Edwards SW: Mcl-1; the molecular regulation of protein function. FEBS Lett 584: 2981-2989, 2010.

40. Zhang J, Yu XH, Yan YG, Wang C and Wang WJ: PI3K/Akt signaling in osteosarcoma. Clin Chim Acta 444: 182-192, 2015.

41. Na YS, Yang SJ, Kim SM, Jung KA, Moon JH, Shin JS, Yoon DH, Hong YS, Ryu MH, Lee JL, et al: YM155 induces EGFR suppression in pancreatic cancer cells. PLoS One 7: e38625, 2012. 